\title{
Non-apoptotic functions of caspase-7 during osteogenesis
}

\author{
E Svandova ${ }^{1,2}$, H Lesot ${ }^{3,4}$, T Vanden Berghe ${ }^{5,6}$, AS Tucker ${ }^{7}$, PT Sharpe ${ }^{7}$, P Vandenabeele ${ }^{5,6}$ and E Matalova ${ }^{*, 1,2}$
}

Caspase-3 and -7 are generally known for their central role in the execution of apoptosis. However, their function is not limited to apoptosis and under specific conditions activation has been linked to proliferation or differentiation of specialised cell types. In the present study, we followed the localisation of the activated form of caspase-7 during intramembranous (alveolar and mandibular bones) and endochondral (long bones of limbs) ossification in mice. In both bone types, the activated form of caspase-7 was detected from the beginning of ossification during embryonic development and persisted postnatally. The bone status was investigated by microCT in both wild-type and caspase-7-deficient adult mice. Intramembranous bone in mutant mice displayed a statistically significant decrease in volume while the mineral density was not altered. Conversely, endochondral bone showed constant volume but a significant decrease in mineral density in caspase-7 knock-out mice. Cleaved caspase-7 was present in a number of cells that did not show signs of apoptosis. PCR array analysis of the mandibular bone of caspase-7deficient versus wild-type mice pointed to a significant decrease in mRNA levels for Msx1 and Smad1 in early bone formation. These observations might explain the decrease in the alveolar bone volume of adult knock-out mice. In conclusion, this study is the first to report a non-apoptotic function of caspase-7 in osteogenesis and also demonstrates further specificities in endochondral versus intramembranous ossification.

Cell Death and Disease (2014) 5, e1366; doi:10.1038/cddis.2014.330; published online 14 August 2014

Caspase-7 belongs to the trio (caspase-3, -6, -7) of executioner cysteinyl aspartate-specific proteases and can also act as a pro-inflammatory factor. Caspase-7 is involved in apoptosis in a number of tissues. ${ }^{1,2}$ Among these, the cleaved (activated) form of caspase-7 has been detected in the primary enamel knot of the first molar, a site where apoptosis takes place. However, caspase-7 was also detected in non-apoptotic areas of differentiating odontoblasts and ameloblasts. ${ }^{3}$ This observation was further emphasised by the fact that caspase-7 knock-out (Casp7 - / - ) mice showed a significant decrease in the amount of enamel deposition in mouse incisors. ${ }^{4}$ Furthermore, non-apoptotic effects of other caspases have also been reported in other tissues, including a role in proliferation of $\mathrm{T}$ cells, in cell-cycle regulation, differentiation of keratinocytes, formation of erythrocytes or platelets, lens development, and skeletal muscle maturation. ${ }^{5}$

Along with its presence in teeth, the activated form of caspase-7 was observed in the forming alveolar processes developing from the mandibular bone of mouse embryos. ${ }^{3}$ In the present work, we investigated the role of caspase-7 in two types of bone; intramembranous bones (mandibular and alveolar) and endochondral bones (front and hind limbs). In the former type, osteoblasts directly differentiate from the condensed mesenchyme, while in the latter a cartilaginous anlage initially forms that is later replaced by bone. ${ }^{6}$ Osteogenesis consists of several successive steps and is regulated by complex molecular interactions, involving products of genes such as bone morphogenetic proteins - Bmps, ${ }^{7}$ fibroblast growth factors - Fgfs, $^{8}$ hedgehog genes, ${ }^{9}$ vascular endothelial growth factor - Vegf, ${ }^{10,11}$ transforming growth factor - Tgf $\beta$ pathway, ${ }^{12}$ and transcription factors such as Msh homeobox - Msx 1, ${ }^{13}$ SRY-box - Sox9, ${ }^{14}$ and runt-related transcription factor - Runx $2 .^{15}$ In this study, the distribution of caspase-7 was investigated during bone development and the effect of caspase-7 deficiency on bone formation was assessed. The findings uncover novel roles of caspase-7 in bone formation, and reveal differences in action dependent on the developmental history of the bone.

\section{Results}

Activation of caspase-7 during the development of intramembranous and endochondral bones

Intramembranous ossification (mandibular and alveolar bones): At embryonic day (ED) 12.5, condensed mesenchymal cells lateral to Meckel's cartilage were in evidence at the site

\footnotetext{
${ }^{1}$ Institute of Animal Physiology and Genetics CAS, v.v.i., Brno, Czech Republic; ${ }^{2}$ Department of Physiology, University of Veterinary and Pharmaceutical Sciences, Brno, Czech Republic; ${ }^{3}$ INSERM UMR 1109, team "Osteoarticular and Dental Regenerative NanoMedicine", Strasbourg, France; ${ }^{4}$ Faculté de chirurgie dentaire, Université de Strasbourg, Strasbourg, France; ${ }^{5}$ Inflammation Research Centre, VIB, Ghent, Belgium; ${ }^{6}$ Department of Molecular Biology, Ghent University, Ghent, Belgium and ${ }^{7}$ Department of Craniofacial Development and Stem Cell Biology, King's College London, London, UK

*Corresponding author: E Matalova, Institute of Animal Physiology and Genetics, CAS v.v.i., Veveri 97, Brno 602 00, Czech Republic. Tel: + 420532290155 ; Fax: + 420541212 988; E-mail: matalova@iach.cz

Abbreviations: Alp, alkaline phosphatase; Ambn, ameloblastin; BMC, bone mineral content; BMD, bone mineral density; Bmp, bone morphogenetic protein; Bmpr, bone morphogenetic protein receptor; BV/TV, bone volume/tissue volume; Casp7-/-, caspase-7 knock-out; Cbfa1, core binding factor alpha; microCT, micro-computed tomography; Dmp1, dentin matrix protein; ED, embryonic day; Fgf, fibroblast growth factor; Fgfr, fibroblast growth factor receptor; Mmp8, matrix metalloproteinase; Msx1, Msh homeobox; P, postnatal day; PARP, poly(ADP-ribose) polymerase; Runx2, runt-related transcription factor; Sox9, SRY-box9; Tgf $\beta$, transforming growth factor $\beta$; TUNEL, terminal deoxynucleotidyl transferase dUTP nick end labelling; Vegf, vascular endothelial growth factor Received 06.3.14; revised 12.6.14; accepted 19.6.14; Edited by M Agostini
} 
of the developing jaw bones, and both the activated form of caspase-7 and osteocalcin, a marker of osteogenesis, were absent (data not shown). The activated form of caspase-7 appeared first at ED13.5 (Figures 1e and f) coincident with osteocalcin expression (Figures $1 \mathrm{n}$ and o), as the bone matrix of the developing mandible started to become apparent (Figure 1a). During further embryonic development (ED15.5 and ED17.5), localisation of the activated form of caspase-7 remained similar (Figures 1g-k). Meckel's cartilage was negative at ED17.5 (Figure 1j), which was similar also at earlier stages (data not shown). The activation of caspase-7 was localised particularly to the cytoplasm and positively correlated with osteocalcin expression (Figures 1p-s). As development progressed (ED15.5 and ED17.5), the size of the mandibular bone increased (Figures 1b and c). Caspase-7 was apparent also
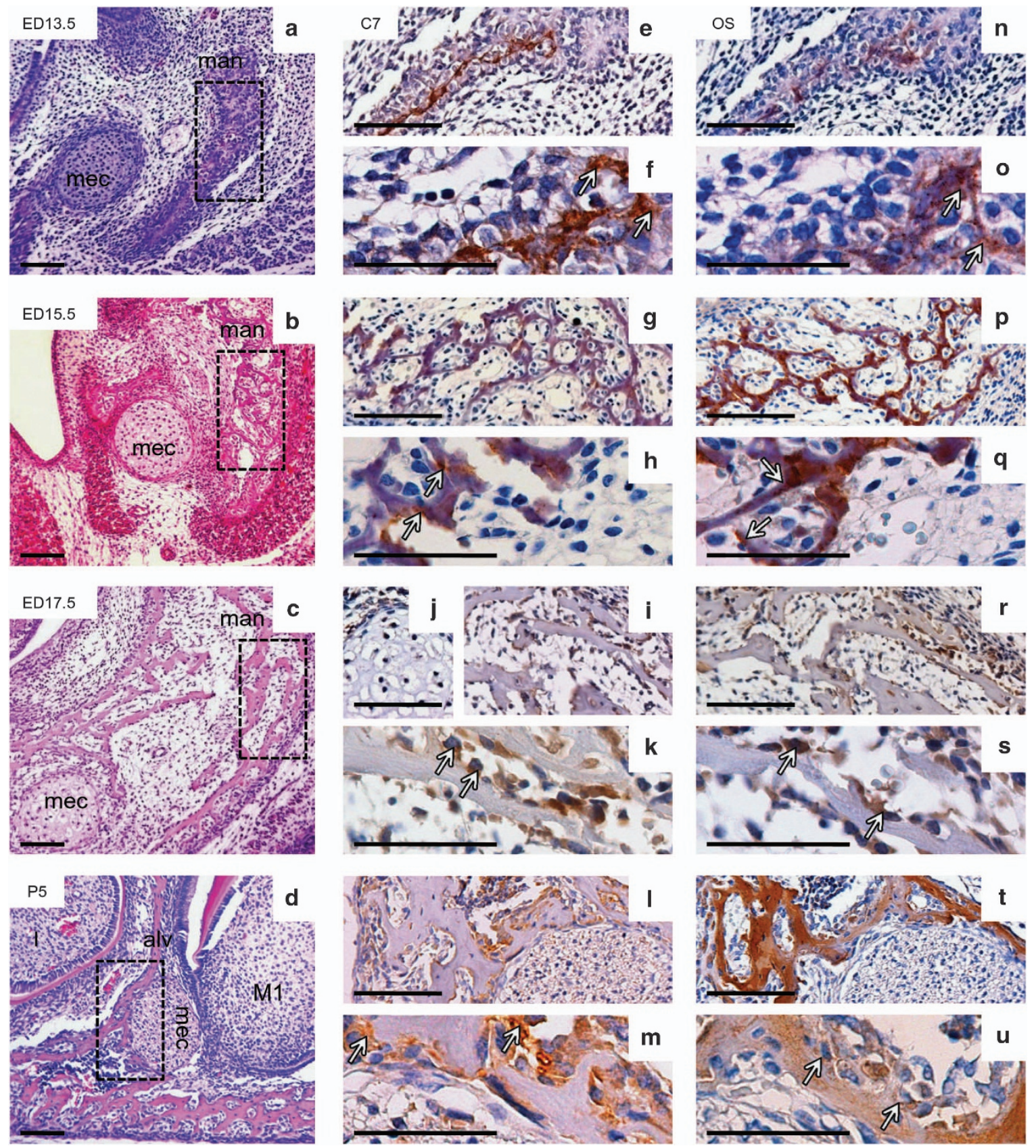

Figure 1 Localisation of activated caspase-7 and osteocalcin in mandibular and alveolar bones at embryonic and postnatal stages. Morphology of mandible visualised by haematoxylin-eosin staining at ED13.5 (a), ED15.5 (b), ED17.5 (c), and P5 (d). Localisation of activated (cleaved) caspase-7 in cells of mandibular bone at ED13.5 (e and f), ED15.5 (g and h), ED17.5 (i-k), and alveolar bone at P5 (I and $\mathbf{~ m ) . ~ E x p r e s s i o n ~ o f ~ o s t e o c a l c i n ~ i n ~ c e l l s ~ o f ~ m a n d i b u l a r ~ b o n e ~ a t ~ E D 1 3 . 5 ~ ( n ~ a n d ~} \mathbf{~})$, ED15.5 (p and $\mathbf{~ q ) , ~ E D 1 7 . 5 ~}$ ( $\mathbf{r}$ and $\mathbf{s}$ ), and alveolar bone at P5 ( $\mathbf{t}$ and $\mathbf{u}$ ). Arrows point to the positive cells (brown); alv (alveolar bone), C7 (caspase-7), I (incisor), M1 (first molar), mand (mandibular bone), mec (Meckel's cartilage), and OS (osteocalcin). Scale bar $=100 \mu \mathrm{m}$ in figures: $\mathbf{a}, \mathbf{b}, \mathbf{c}, \mathbf{d}, \mathbf{e}, \mathbf{g}, \mathbf{i}, \mathbf{j}, \mathbf{l}, \mathbf{n}, \mathbf{p}, \mathbf{r}, \mathbf{t} ;$ scale bar $=50 \mu \mathrm{m}$ in figures: f, h, k, m, o, q, s, u 
at postnatal day (P) 5 in the alveolar bone (Figures 1 and $\mathrm{m}$ ) and still synchronised with osteocalcin expression (Figures 1t and $\mathrm{u}$ ). The precursor cells of the alveolar bone originate from the dental follicle. ${ }^{16}$ During early postnatal development, the alveolar bone forms and becomes a part of the mandible (Figure 1d). While osteocalcin was observed in the cytoplasm of bone cells and also in bone matrix, the activated form of caspase-7 was observed predominantly in the cytoplasm at all stages studied.
Endochondral ossification (bones of front limbs): At ED13.5, neither the activated form of caspase-7 nor osteocalcin was detected in the cartilaginous ulna and radius of the developing forelimb, agreeing with the fact that no bone tissue was visible at this stage (data not shown). When bone formation started, at ED14.5 (Figure 2a), the activated form of caspase-7 was detected in a thin cellular layer (periosteum) adjacent to the surface of hypertrophic chondrocytes (Figures 2e and f). Osteocalcin was detected
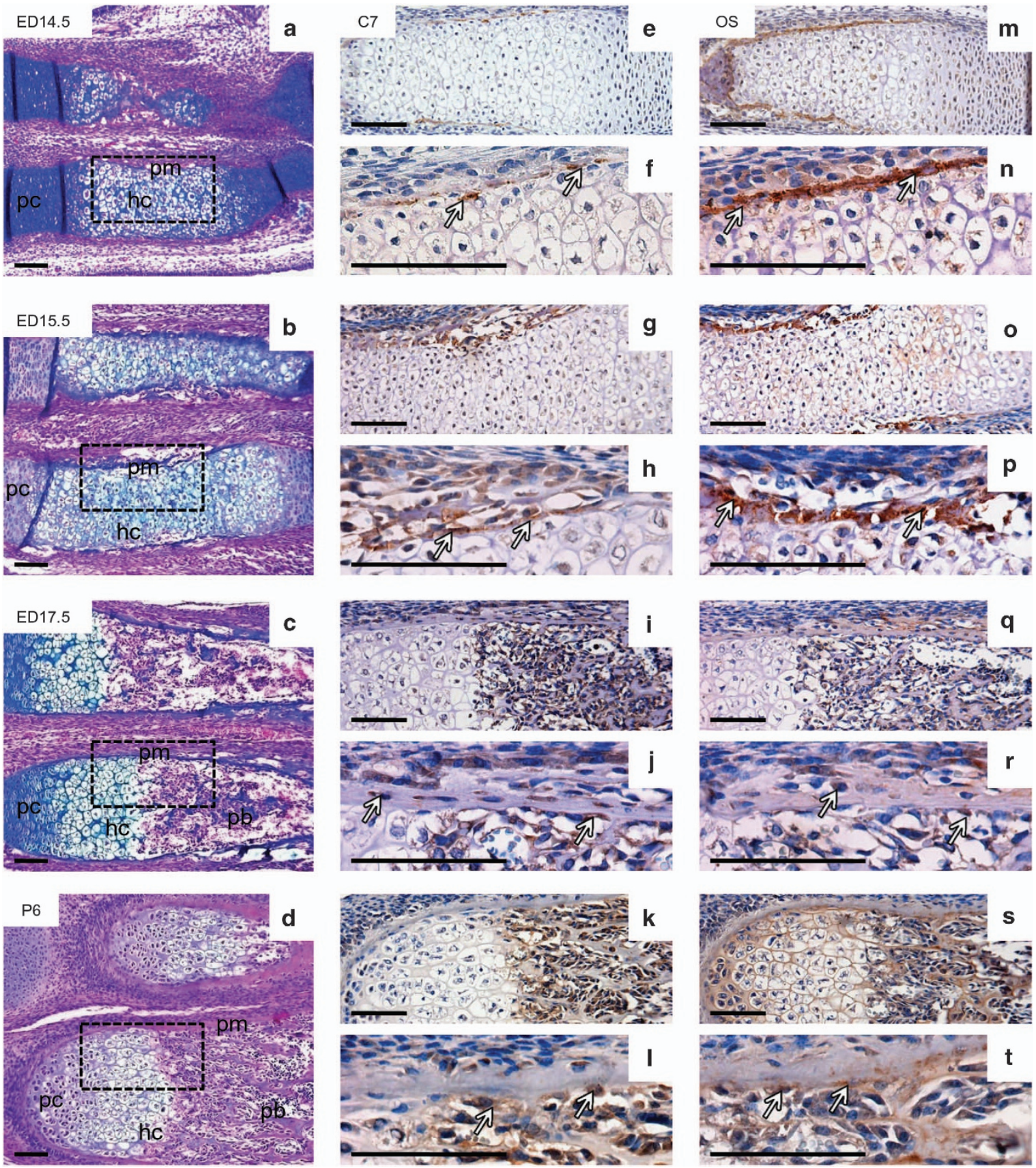

Figure 2 Localisation of activated caspase-7 and osteocalcin in long bones of front limb (ulna, radius) at embryonic and postnatal stages. Morphology of bones of front limb visualised by haematoxylin-eosin and Alcian blue staining at ED14.5 (a), ED15.5 (b), ED17.5 (c), and P6 (d). Localisation of activated (cleaved) caspase-7 in areas of ossification at ED14.5 (e and f), ED15.5 ( $\mathbf{g}$ and h), ED17.5 (i and j), and P6 ( $\mathbf{k}$ and $\mathbf{l})$. Expression of osteocalcin in areas of ossification at ED14.5 ( $\mathbf{m}$ and $\mathbf{n})$, ED15.5 (o and p), ED17.5 (q and r), and P6 (s and t). Arrows point to the positive cells (brown); C7 (caspase-7), hc (hypertrophic chondrocytes), OS (osteocalcin), pb (primary bone), $\mathrm{pc}$ (proliferating chondrocytes), and pm (perichondrium). Scale bar $=100 \mu \mathrm{m}$ 
at the same time and spatially co-distributed with caspase-7 (Figures $2 \mathrm{~m}$ and $\mathrm{n}$ ). Later, at ED15.5 (Figures $2 \mathrm{~g}$ and $\mathrm{h}$ ), caspase-7 was visualised in the enlarging zone of ossification, at ED 17.5 (Figures 2i and j) caspase-7 was localised in the region of the bone collar. A similar pattern was observed for osteocalcin (Figures 2o-r). During this period (ED15.5-ED17.5), the size of the bone tissue progressively increased (Figure 2b). From ED17.5, proliferating, prehyperthrophic, and hyperthrophic chondrocytes as well as primary bone were clearly detectable (Figure 2c). Finally, at P6, caspase-7 (Figures 2k and I) and osteocalcin (Figures 2s and $t$ ) were detected in forming primary bone (Figure $2 d$ ). The proliferating and hypertrophic zones of the cartilage were caspase-7 negative at all stages examined. As observed in the alveolar bone, the activated form of caspase-7 was detected particularly in the cytoplasm at all stages.

Correlation between caspase-7-positive cells and apoptosis in the alveolar bone. Apoptosis is associated with bone remodelling during postnatal stages of tooth and jaw development. ${ }^{17}$ At P0, apoptosis was observed in the alveolar bone (Figure $3 \mathrm{~b}$ ), where activated caspase-7 was also present (Figure 3a). Not all caspase-7positive cells, however, were associated with the terminal deoxynucleotidyl transferase dUTP nick end labelling (TUNEL)-positive regions. In some TUNEL-positive cells, the nucleus showed positive staining for active caspase-7 (Figures $3 \mathrm{a}$ and $\mathrm{b}$ ), indicating that apoptosis was working through this caspase, which was also documented in our previous study on primary enamel knot. ${ }^{3}$ However, in those cells with active caspase-7 in the cytoplasm no TUNEL staining was observed, indicating a dual function for caspase-7 depending on its subcellular localisation (Figures $3 a$ and $b$ ).

Influence of caspase-7 deficiency on intramembranous and endochondral bone formation in adult mice morphological aspects

Alveolar bone: The length of molar row and cusps pattern were not altered in caspase-7-deficient mice (Figure 4d) compared with wild types (Figure 4a), as observed from 3D reconstructions of micro-computed tomography (microCT) images. Sagittal and frontal sections in the molar region of the jaw did not reveal any striking difference in the alveolar bone organization in Casp 7-/ - mice (Figures $4 \mathrm{e}$ and f) compared with wild type (Figures $4 \mathrm{~b}$ and $\mathrm{c}$ ).
Femur: 3D reconstruction of the distal femur showed different organisation of trabeculae in Casp7-/- mice, as observed in longitudinal (Figure 5f) or transversal (Figure 5e) sections, when compared with wild-type mice (Figures 5b and a). Changes in endosteal (Figure $5 \mathrm{~g}$ ) and periosteal (Figure $5 \mathrm{~h}$ ) surfaces were also recognised in Casp7 - / - mice, where a smoothed surface was observed, in contrast to the greater porosity observed in the cortex in wild-type mice (Figures 5c and d).

\section{Influence of caspase-7 deficiency on intramembranous and endochondral bone formation of adult mice - quantitative analysis}

Alveolar bone (intramembranous): MicroCT analysis of the alveolar bone of the mandible in Casp7 - / - mice showed a statistically significant $(P=0.024)$ decrease (Figure $6 \mathrm{~b})$ in bone mineral content (BMC). The bone mineral density (BMD), however, was not decreased (Figure 6c). Interestingly, the bone volume in Casp7 - / - mice appeared to be significantly $(P=0.024)$ decreased (Figure 6a) which was not clearly apparent from the morphological assessment. These results point to an overall decrease in alveolar bone volume. The decrease in alveolar bone volume was estimated to be around $24 \%$. Therefore, reduction in one dimension would appear to be $<3 \%$, which is difficult to recognise by morphological assessment. Similar trends were obtained by examination of the whole mandible (data not shown).

Femur (endochondral): In femoral trabecular bone of Casp7 - I- mice, microCT analysis revealed a statistically significant $(P=0.009)$ decrease in BMC (Figure 6f) and a significant $(P=0.009)$ decrease in BMD (Figure 6g). Analysis of the cortex also showed a significant $(P=0.011)$ decrease in BMC (Figure 6d) and a significant $(P=0.009)$ decrease in BMD (Figure 6h). In contrast to the alveolar bone, the volume of bone was not reduced (Figure $6 \mathrm{e}$ ) and the bone volume/ tissue volume (BV/TV) ratio (Figure 6i) was unaffected $(P=0.886)$. Trabecular separation and thickness were not impacted by caspase-7 deficiency (data not shown).

Caspase-7 versus caspase- 3 in bone. To assess whether any other caspases compensated for the loss of caspase-7, we analysed the activation of the effector caspase, caspase-3. Immunohistochemical analysis of mandibular bone at stage ED 15.5 (Figures $7 a$ and $b$ ) did not show any apparent increase in activation of caspase- 3 in the caspase-7-deficient mandibular bone (Figures 7c and d) compared with wild type
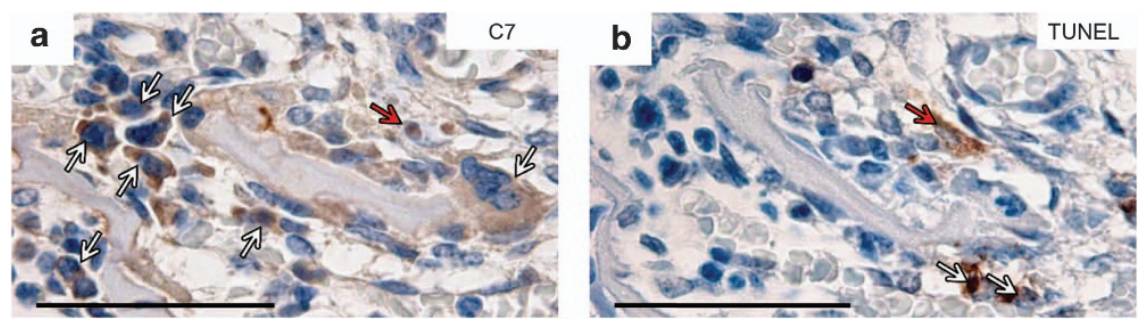

Figure 3 Co-localisation of caspase-7-positive cells and apoptosis in alveolar bone at P0. Localisation of activated (cleaved) caspase-7 in cells of alveolar bone (a), apoptotic cells visualised by TUNEL assay (b). White arrows point to the positive cells (brown), red arrows point to apoptotic cell (b) and cell with nuclear localisation of caspase-7 (a); C7 (caspase-7). Scale bar $=50 \mu \mathrm{m}$ 

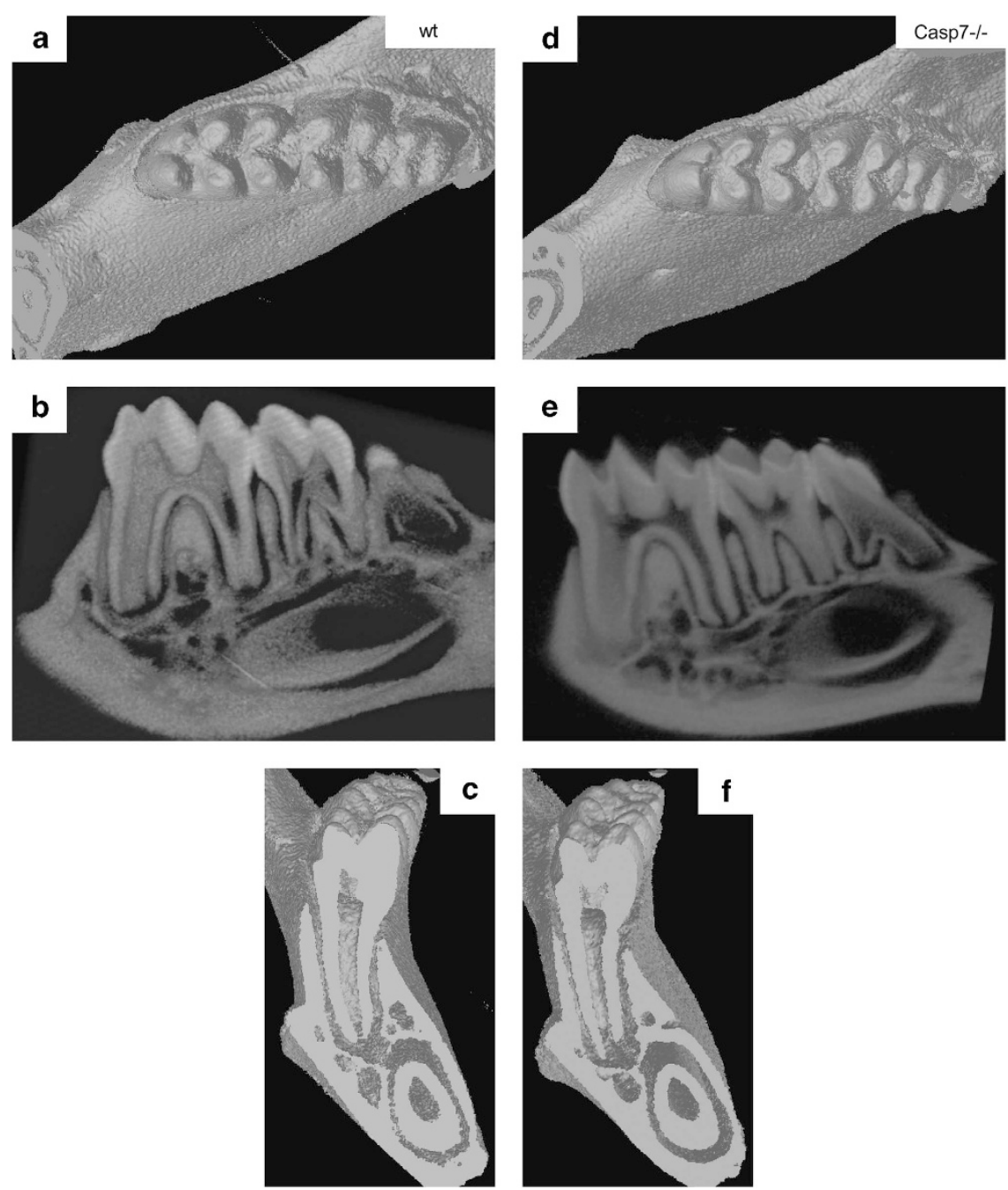

Figure 4 Morphological evaluation of alveolar bone in adult Casp7 - / - and wild-type mice. Row of three mice molar in wild type and (a) and Casp7 - / - (d), sagittal section of mandible in wild type (b) and Casp7 - I- (e), frontal section of the middle part of the first molar region in wild type and (c) and Casp7 - I- (f); wt (wild type)

(Figures 7e and f). In keeping with this finding, the distribution of caspase-7 did not always overlap with that of caspase-3 (see Figures 7o and p). In addition, we observed no decrease in the distribution of apoptotic cells in caspase-7-deficient mice (Figures $7 \mathrm{~g}-\mathrm{j}$ ), nor in the expression of osteocalcin (Figures $7 \mathrm{k}-\mathrm{n}$ ). At P0, activation of caspase-3 and -7 was found in a similar number of osteoclasts (activated caspase-3 was detected in $54 \%$ of osteoclasts and activated caspase- 7 was detected in $50 \%$ of osteoclasts). However, activation of both caspases was different in osteoblasts (activated caspase- 3 was detected in $28 \%$ of osteoblasts and activated caspase- 7 was detected in $71 \%$ of osteoblasts). Overall, our data indicate that caspase- 3 probably does not compensate for the loss of caspase-7 as a general mechanism during bone formation.

Impact of caspase-7 deficiency on osteogenic gene expression. Gene expression changes in isolated mandibular bone were assessed using a PCR array comprised of 84 genes involved in osteogenesis
(Figures 8a-c). Caspase-7-deficient mandibular bone showed a statistically significant decrease in the expression of Msx1 (fold change: $-2.17 ; P=0.0058$ ) and Smad1 (fold change: $-2.2 ; P=0.0147$ ) in early development (ED15.5). In contrast, an increase in liver/bone/kidney form of alkaline phosphatase - Alp (fold change: $2.14 ; P=0.0438$ ) was found in the Casp7 - / - mandibular bone. Moreover, a decreasing trend but not a statistically significant drop was observed in other important genes implicated in ossification such as ameloblastin - Ambn (fold change: - 2.99), bone morphogenetic protein receptor - Bmpr1a (fold change: -2.18 ), dentin matrix protein - Dmp1 (fold change: - 4.64), Fgf2 (fold change: -2.29 ), fibroblast growth factor receptor Fgf2r (fold change: - 2.02), matrix metalloproteinase Mmp8 (fold change: -3.21 ).

\section{Discussion}

Caspases have been described as taking part in both apoptotic and non-apoptotic processes. ${ }^{18,5}$ Previous 

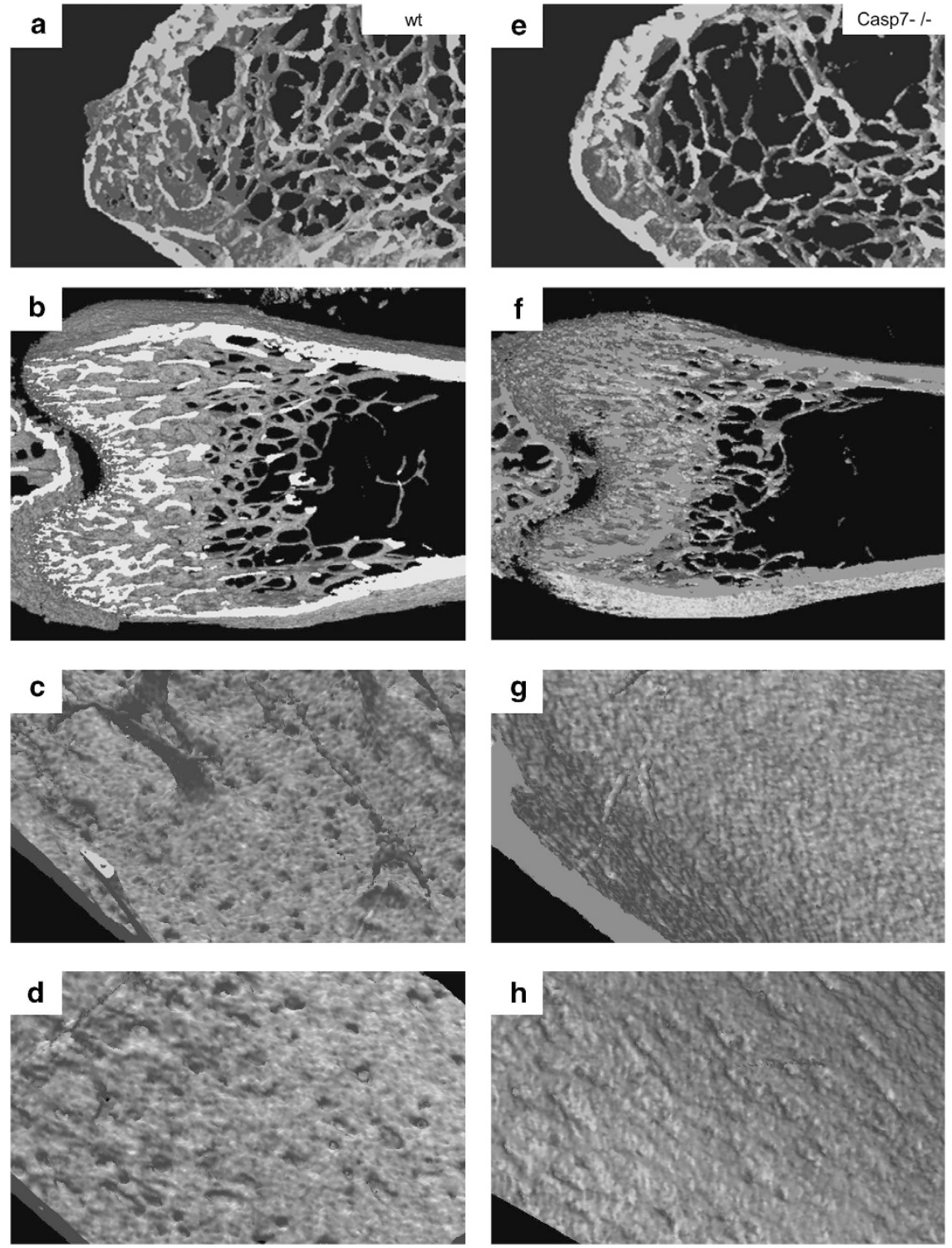

Figure 5 Morphological evaluation of distal femur in adult Casp7 - / - and wild-type mice. Transversal section of femur in wild type (a) and Casp7 - / - (e), longitudinal section of wild type (b) and Casp7 - / - (f), endosteal surface of wild type (c) and Casp7 - / - (g), periosteal surface of wild type (d) and Casp7 - / - (h); wt, wild type

investigations have shown that caspases have a role in cell differentiation either involving incomplete apoptosis (e.g., cells of the lens) or by completely non-apoptotic pathways (macrophages and skeletal muscles). ${ }^{19}$ With reference to non-apoptotic functions, caspase-7 was proposed to be involved in hard tissue formation, ${ }^{4}$ as immunostaining for caspase-7 showed its presence in developing mandibular bone. ${ }^{3}$ This observation led us to investigate the localisation of caspase-7 in cells of the intramembranous bones of the mandible from ED12.5 to P5 and the bone phenotype in adult (P28) Casp7 - / - mice.

In the present work, the activated form of caspase-7 was detected in the mandibular bone from early stages of development (ED13.5). At this stage, the developing mandibular bone can be identified as emerging from a mass of condensed mesenchymal cells. ${ }^{20}$ Activated caspase- 7 was present at the same time as osteocalcin, used as a marker for osteogenesis. This pattern for the activated form of caspase-7 in mandibular bone, and later also in alveolar bone, persisted at postnatal stages. Caspase-7-positive cells predominated in non-apoptotic regions. This activation pattern therefore indicates that caspase- 7 might have a role in both early bone formation and later bone development.

Analysis of Casp7-/ - mice showed that the BMD was comparable to that of wild-type animals. However, the BMC and volume of the alveolar bone were significantly decreased, when compared with wild types suggesting an overall decrease in alveolar bone volume. Similar trends were obtained also by examination of the mandible. Thus, data from immunohistochemistry and microCT analysis pointed to the role of caspase-7 in the formation of the alveolar bone.

The bones of the lower jaw form by intramembranous ossification, as a comparison we therefore also investigated long bones, which form by endochondral ossification. 
a

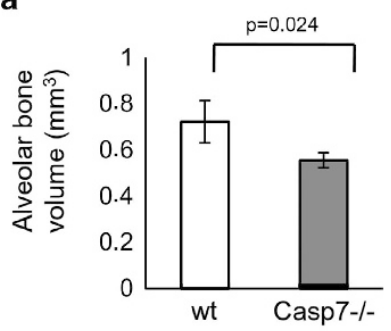

b

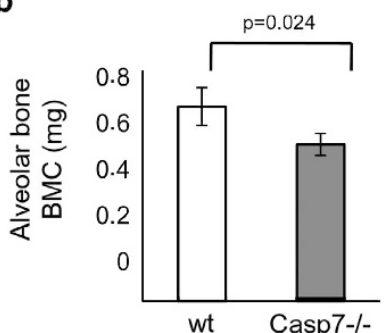

C

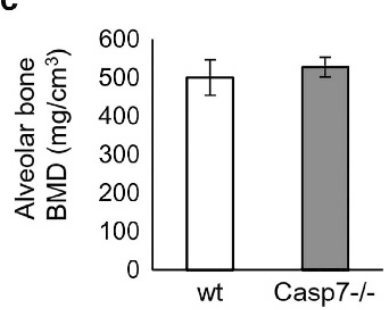

f

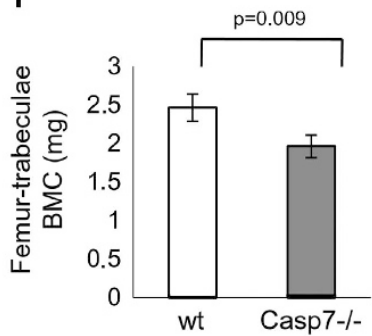

g

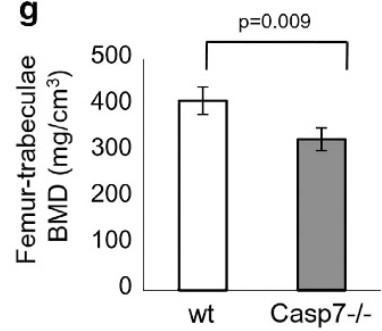

d

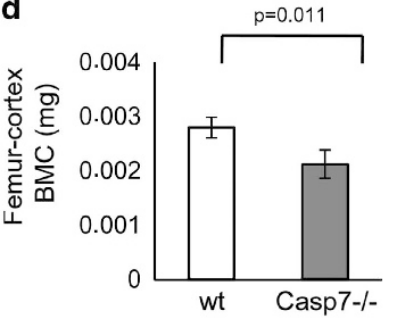

h

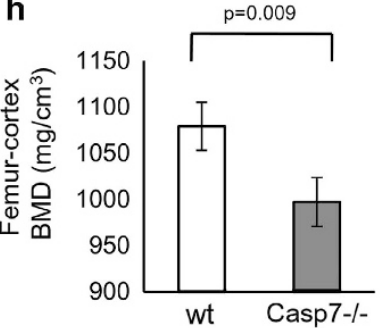

i

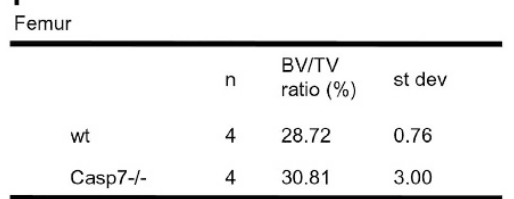

Figure 6 MicroCT analysis of bone parameters of alveolar bone and femur in Casp7 - / - and wild-type mice. Bone volume of alveolar bone (a) and femur (e), BMC of alveolar bone (b) and femur (f), BMD of alveolar bone (c) and femur (g), BMC of femoral cortex (d), BMD of femoral cortex (h), and BV/TV ratio of femur (i); wt (wild type)

The activated form of caspase-7 was detected in long bones throughout embryonic and early postnatal development. The first time point of detection was ED14.5, when the centres of ossification initiate in the mouse front limbs. ${ }^{21}$ The timing of initiation of active caspase-7 in mandibular and long bone reflects the distinct timing of ossification in both models. A similar delay of 1 day between the jaw primordia (ED11.5) and femur (ED12.5) has also been reported for the expression of Core binding factor alpha - Cbfa1 (Runx2), a marker of osteoblast differentiation. ${ }^{21}$
MicroCT analysis showed differences in bone organisation, as the trabeculae system was decreased and changes in endosteal and periosteal surfaces were apparent in Casp7 - / - mice. In contrast, microCT analysis showed that parameters such as trabecular thickness and separation, bone volume, or BV/TV ratio were not altered in Casp7 - / mice. However, a significant decrease in BMC and BMD was detected in both cortical and trabecular bones.

The effect of caspase-7 deficiency, as observed by microCT, was quite distinct in intramembranous and endochondral bones. One explanation for this may relate to the different characteristics of both types of ossification. ${ }^{22}$ Developmental differences in intramembranous and endochondral ossification have been reported when comparing gene expression patterns of periostin, ${ }^{23} \mathrm{Bmps}^{24}$ or variation in the vascularisation. ${ }^{25}$ Furthermore, deficiency of Bmp2/6 resulted in reduced endochondral bone formation, but had no impact on intramembranous bone. ${ }^{26}$ Therefore, the expression or/and function of distinct genes in both models may differ, which may also be the case for caspase-7.

During mineralisation of the long bone, caspase-7 as a protease may be involved in specific transcription factor cleavage, ${ }^{27}$ which might induce a specific cell lineage programme in bone cells. Caspase-7 does not, however, seem to be a critical factor for early stages of bone formation. Our studies show decreased bone density in the femur and decreased volume of alveolus in adult Casp7 - / - mice.

Structural similarities ${ }^{28}$ and tissue-specific functional redundancies between caspase- 7 and caspase- 3 have previously been reported. ${ }^{1,3,29}$ In contrast to the relatively mild effect of caspase-7 deficiency in the femur, caspase-3deficient mice showed a reduction in trabecular thickness and decreased BV/TV ratio. ${ }^{30}$ In Casp7 - / - mandibular bone, an increase in the activation of caspase-3 was not observed compared with wild-type mice. Moreover, the expression of activated caspase-3 and caspase-7 did not overlap completely in wild-type tissue, with activated caspase-7 being detected in a larger number of osteoblasts compared with caspase-3. We therefore conclude that the relatively mild bone phenotype we describe in the caspase-7 knock-outs was not due to compensation by caspase-3. Previous studies also delineated partial differences in the protease activities, ${ }^{31}$ in substrate specificity and localisation of the two activated caspases as described in murine liver after Fas stimulation. ${ }^{32,33}$ From these observations, caspase-3 appeared more effective in the cleavage of molecules with low molecular weight, ${ }^{31,34}$ and was also more promiscuous. ${ }^{32}$ Therefore, caspase-7 in the femur may have a much more specific effect on bone mineralisation. The importance of caspases in bone formation is also supported by studies where overexpression of $\mathrm{BCl}-2$ was shown to result in the inhibition of osteoblast differentiation. ${ }^{35}$

To try to better understand the effect of caspase-7, osteogenic signalling was investigated in Casp7 - /- and wild-type mice. PCR array analysis of genes involved in osteogenesis was performed on total RNA obtained from the mandibular bone (including also developing alveolar processes) at ED15.5. At this early stage of mandibular bone formation in caspase-7-deficient mice, several genes involved in osteogenesis (Bmpr1a, Ambn, Dmp1, Fgf2, Fgfr2, and 

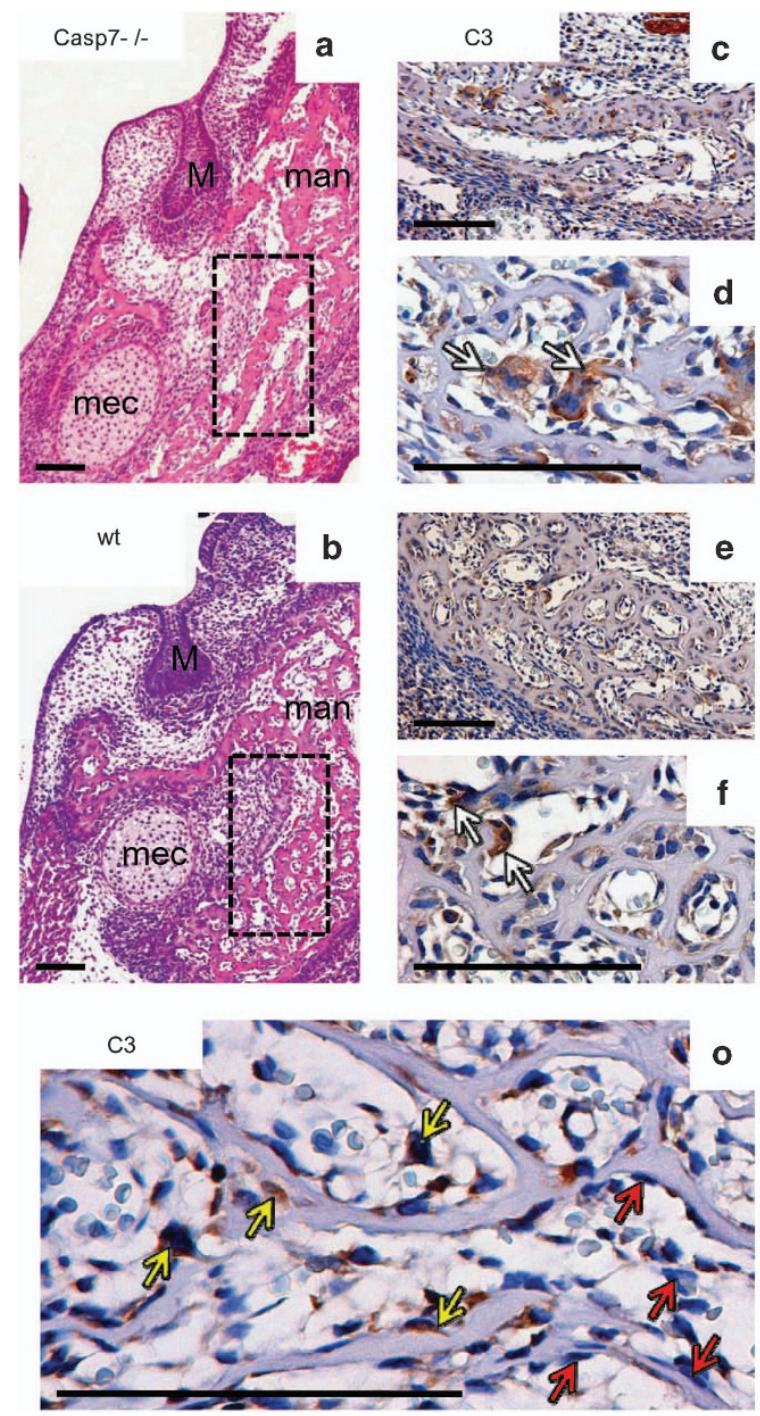
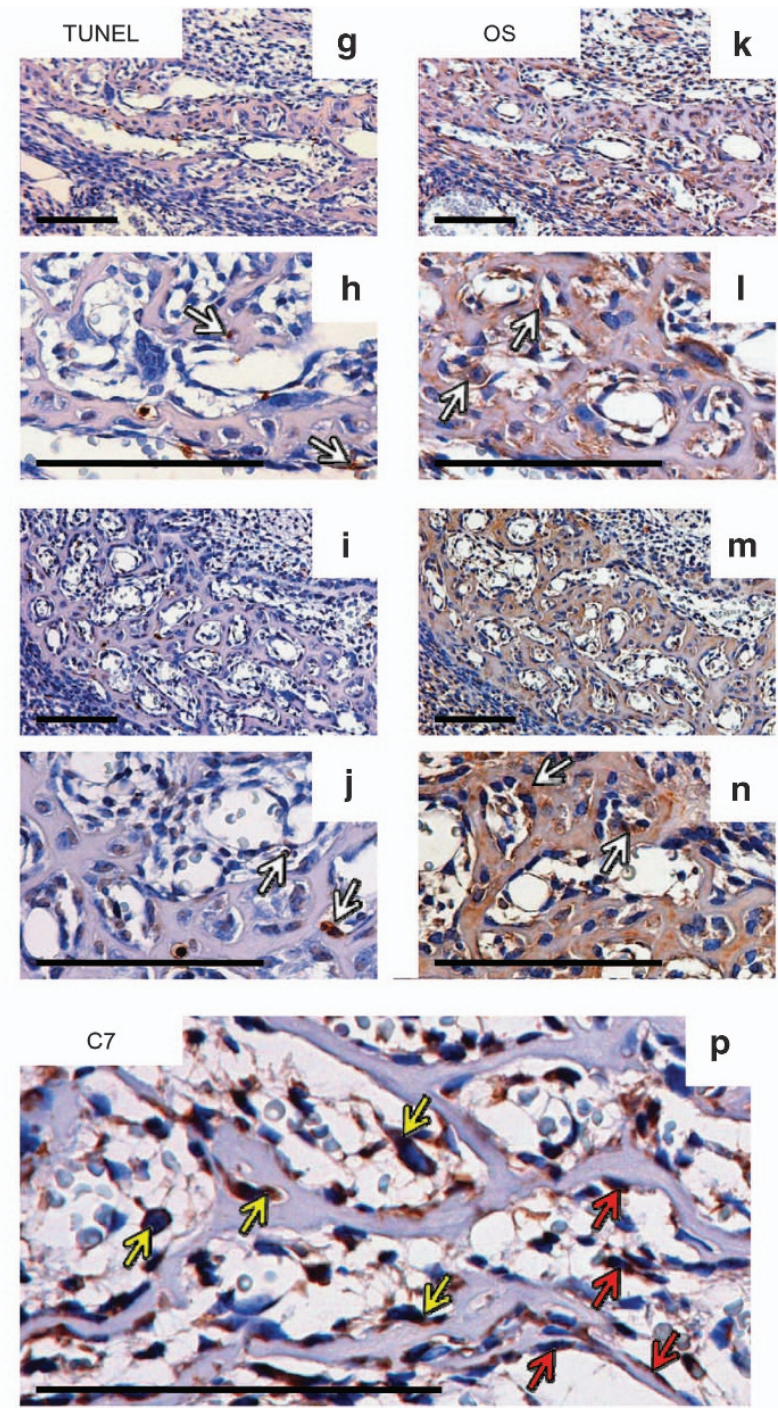

Figure 7 Activation of caspase-3 (c-f), distribution of apoptotic cells $(\mathbf{g}-\mathbf{j})$, and expression of osteocalcin (k-n) in Casp7 - / - (a, c, d, g, h, k, l) versus wild-type mice $(\mathbf{b}, \mathbf{e}, \mathbf{f}, \mathbf{i}, \mathbf{j}, \mathbf{m}, \mathbf{n})$ at ED15.5, and spatial correlation of caspase-3 (o) and caspase-7 (p) in wild-type mice at P0. Morphology of mandible visualised by haematoxylin-eosin staining in Casp7 - I - (a) and wild-type mice (b). White arrows point to the positive cells (brown), yellow arrows point to cells with activation of caspase-3 and caspase-7, red arrows point to cells where solely caspase-7 is activated; C3 (caspase-3), C7 (caspase-7), man (mandibular bone), mec (Meckeĺs cartilage), OS (osteocalcin). Scale bar $=100 \mu \mathrm{m}$

Mmp8) showed a decreasing trend, although this was not statistically significant. All these genes are involved in different periods of bone formation. . $^{7,836-38}$

Moreover, there was a statistically significant decrease in Msx1 and Smad1, both of which are involved in bone formation. ${ }^{12,39}$ Smad1 is one of the key players in BMP pathway and induces bone formation. ${ }^{40}$ In vitro studies point to a role for Smad1 in osteoblasts terminal differentiation ${ }^{41}$ and inhibition of $B m p 6$, which acts upstream of Smad1, results in the inhibition of osteogenesis in alveolar bone ${ }^{42}$ Moreover, a decrease in BMP signalling has been suggested to result in a decreased bone volume in young adult mice. ${ }^{43}$

Therefore, the reduced levels of Smad1 and Msx 1 products during embryonic development support the significant decrease in intramembranous bones volume that we observed in adult Casp7 - / - mice.

Despite a significant decrease in the volume of the alveolar bone in adult Casp7 $-/-$ mice, PCR arrays revealed a significant increase in Alp mRNA in Casp7-/- mice at ED15.5. ALP is present at the surface of osteoblasts and its activity is required for production of high phosphate concentration for bone mineralization. ${ }^{44}$ Previous investigations described a positive correlation between Alp mRNA levels and ALP activity although contradictory results also exist. ${ }^{45-47}$ Notably, higher levels of ALP activity were associated with lower levels of Alp mRNA. ${ }^{48}$ ALP activity cannot thus be determined from Alp mRNA synthesis. There may be several post-transcriptional/post-translational modifications involved in production of active ALP by osteoblast.

In conclusion, this study of Casp7 - / - mice shows for the first time that the presence of caspase-7 influences intramembranous and endochondral bone development in specific ways. Previous studies have shown that executive caspases are cleaved/activated in the cytoplasm. ${ }^{33,49,50}$ Afterwards, they are translocated to the nucleus to perform their function by cleavage of poly(ADP-ribose) polymerase - PARP or other 


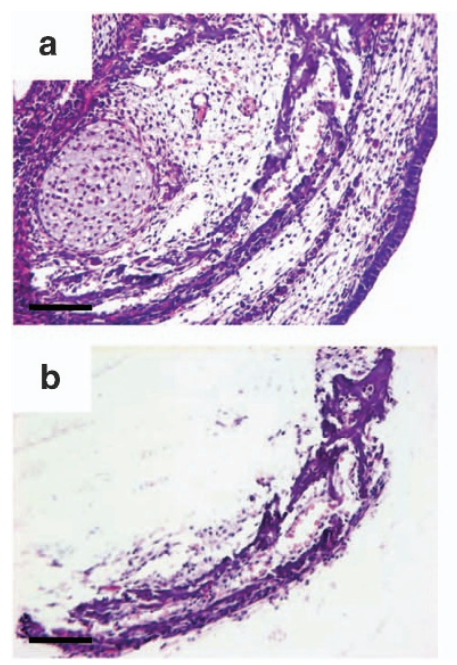

\section{C}

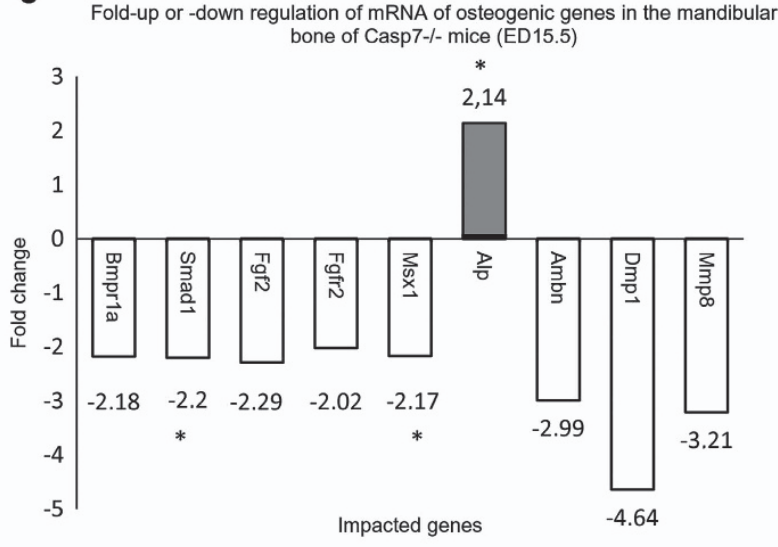

Figure 8 Changes of osteogenic gene expression in Casp7 - / - and documentation of mandibular bone separation for PCR array analysis. Frontal section of murine head (ED15.5), haematoxylin-eosin (a), mandibular bone cut off the surroundings under stereoscopic conditions, haematoxylin-eosin (b). Examination of osteogenic gene expression in Casp7 - I - by PCR array (c), significant changes are marked by asterisk. Scale bar $=100 \mu \mathrm{m}$

proteins such as chromosomal passenger complex members. ${ }^{51-53}$ In the present study focusing on bone, caspase-7 was not detected in the nucleus. Moreover, the mandibular bone in the caspase-7 knock-out did not show any apparent decrease in the distribution of apoptotic cells. These observations further support the non-apoptotic functions described above. A similar situation with a different context has already been reported for $\beta$-catenin. ${ }^{54}$ Our study highlights the fact that the nuclear or cytoplasmic localisation of caspase-7 may impact on its mode of function (cell death, inflammation, growth regulation, or differentiation), and it will be important to investigate this further to try and understand what determines subcellular localisation.

\section{Materials and Methods}

Samples. Mouse heads and front limbs at ED 12.5, 13.5, 14.5, 15.5, 17.5 and P 5,6 were used to investigate localisation of activated form of caspase-7 in bone formation. Collected samples were fixed in $4 \%$ buffered paraformaldehyde for $24 \mathrm{~h}$. For immunohistochemistry procedure dehydrated in ethanol gradient, treated with xylene, and embedded in paraffin. Postnatal mouse heads or front limbs at embryonic stages ED17.5 were decalcified in buffered EDTA before dehydration. Frontal sections of heads and longitudinal sections of front limbs were prepared. Mice were killed according to the experimental protocol 37/2011G approved by Department of Physiology, UVP, Brno, Czech Republic.

Casp7 - I - mice that had been backcrossed over at least 10 generations were provided by Professor $P$ Vandenabeele ${ }^{55}$ and samples processed as for wild-type animals. Embryonic (ED15.5) and postnatal (P28) stages of caspase-7-deficient mice and their littermates were involved in the study.

Immunohistochemistry. Slides were treated with xylene to remove the paraffin, rehydrated through an ethanol series and pre-treated in citrate buffer $(\mathrm{pH}=6.0) 15 \mathrm{~min} / 98^{\circ} \mathrm{C}$. Endogenous peroxidase activity was eliminated by $3 \%$ hydrogen peroxide in PBS $(5 \mathrm{~min} / \mathrm{room}$ temperature). The primary antibodies (9664-Cleaved caspase-3 and 9491S-Cleaved caspase-7, Cell Signaling, Danvers, MA, USA; ab93876-Osteocalcin, Abcam, Cambridge, UK) were diluted 1:50 (caspase-7) and 1:100 (Osteocalcin) and applied overnight $/ 4{ }^{\circ} \mathrm{C}$, peroxidase-conjugated streptavidin-biotin system (Vectastain, Burlingame, CA, USA) and chromogen substrate diaminobenzidine (DAB, K3466, Dako, Carpinteria, CA, USA) reactions were used to visualise positive cells as brown. Slides were counterstained by haematoxylin to clearly distinguish the cell nuclei. Negative control was performed by omitting the primary antibody, specificity of anti-caspase antibodies was verified using caspase-7-deficient tissues. Counting of caspase-3- and caspase-7-positive cells was carried out under magnification $\times 1000$ (for osteoclast, $n=50$; for osteoblasts, $n=500$ ).

TUNEL assay. Slides were treated with xylene, rehydrated in ethanol series, and endogenous peroxidase was blocked by 3\% hydrogen peroxide in PBS ( $5 \mathrm{~min} / \mathrm{room}$ temperature). Samples were pre-treated with proteinase $\mathrm{K} 20 \mathrm{mg} / \mathrm{ml}$ (15 min/room temperature). The reaction mixture (TUNEL, S7100, Millipore, Billerica, MA, USA) was prepared as follows: $3 \mu$ l TdT enzyme, $42 \mu$ ldistilled water, $105 \mu \mathrm{l}$ reaction buffer, which was incubated for $45 \mathrm{~min}$ at $37^{\circ} \mathrm{C}$. An antidigoxigenin-peroxidase reaction was performed for $30 \mathrm{~min} / \mathrm{room}$ temperature. Positive cells were finally visualised by the chromogenic substrate diaminobenzidine (DAB, K3468, Dako). Samples were counterstained by haematoxylin.

MicroCT analysis. Specimens for microCT were scanned using a GE Locus SPmicroCT scanner (GE Healthcare, London, ON, Canada). The specimens were immobilised using cotton gauze and scanned to produce $1 \mu \mathrm{m}$ voxel size volumes, using a X-ray tube voltage of $80 \mathrm{kVp}$ and a tube current of $80 \mu \mathrm{A}$. An aluminium filter $(0.05 \mathrm{~mm})$ was used to adjust the energy distribution of the $X$-ray source. $\mathrm{CT}$ attenuation values were calibrated to hydroxyapatite standards. In femur, bone parameters were assessed in the distal part, the region of interest was restricted under the growth plate extending $2 \mathrm{~mm}$ proximally. Two bone compartments (cortex and trabeculae) were separated by manual contouring that was outlined adjacent to endocortical boundary. Alveolar bone was analysed as one domain. Regions of interest were reconstructed and analysis of BMC, BMD, BV, and TV was carried out using the Microview 2.2 software package (GE Healthcare).

The decrease in alveolar bone was calculated from the average values (four samples in both groups) of bone volume analysed by microCT. When compared with wild type (determined as $100 \%$ volume), the alveolar bone volume of caspase7-deficient mice was calculated as $76 \%$. Therefore, the decrease was $24 \%$.

3D images were post processed by the VGStudio MAX 2.2 software (VGStudio MAX, Heidelberg, Germany). Statistical analysis was evaluated by Sigma Plot 11.2 (Systat software $\mathrm{GmbH}$, Erkrath, Germany). T-test $(P<0.05)$ was determined for assessment of statistical significance in all cases except BV/TV ratio, where Mann-Whitney test was used. Eight adult specimens were analysed in both alveolar bone and femur (four wild types and four Casp7 - / - ).

RNA isolation. Before the processing, mice heads were stored in RNA Later, washed in PBS and fixed in $4 \%$ paraformaldehyde up to $24 \mathrm{~h}$. Samples were dehydrated in ethanol gradient, treated with xylene, embedded in paraffin and $4 \mu \mathrm{m}$ slices were prepared. Samples embedded in paraffin were arranged during 1 week. Mandibular bones were accurately cut off each slice under stereoscopic conditions as documented in Figures $7 a$ and $b$. Selected parts of tissue were used for RNA isolation by the RNeasy FFPE Kit (Qiagen, Valencia, CA, USA). All procedures were carried out in RNAse-free conditions. 
PCR array. Expression of gene panel connected with osteogenesis was assessed by PCR array. Examination of 84 genes was performed in the assay. Total mRNA was transcribed into CDNA and used for the investigation by Mouse Osteogenenic PCR array (PAMM-026A-24, SA Biosciences, Frederick, MD, USA). Data were statistically evaluated by PCR array Data Analysis V4 (SA Biosciences). Statistical significance was determined using a $t$-test $(P<0.05)$. The threshold of fold change was established as $-2 / 2$ fold-change, that means decrease/increase of $100 \%$ in investigated sample. Six embryonic specimens (ED15.5) were analysed (3 wt, 3 Casp7 - / - ).

\section{Conflict of Interest}

The authors declare no conflict of interest.

Acknowledgements. The research was funded by the Grant Agency of the Czech Republic P502/12/1285 at the UVPS (caspase research) and P302/12/J059 at the IAPG CAS, v.v.i. (tooth-bone research). International collaboration runs under Academy of Sciences (project M200451201) and the Brno labs under RVO: 67985904. ES was supported by EMBO ASTF 372-2013. We also acknowledge Chris Healy for help with microCT analysis, Marcela Buchtova for PCR Array consultations, Barbora Vesela for technical support and Bernard Senger for help with statistics. TVB holds a postdoctoral fellowship from the FWO. Research in the Vandenabeele group is funded by European grants (FP6 ApopTrain, MRTN-CT035624; FP7 EC RTD Integrated Project, Apo-Sys, FP7-200767; Euregional PACT II), Belgian grants (Interuniversity Attraction Poles, IAP 7/32), Flemish grants (Research Foundation Flanders, FWO G.0875.11, FWO G.0973.11 and FWO G.0A45.12N, FWO G.0787.13N, Methusalem), Ghent University grants (MRP, GROUP-ID consortium), grant from the Foundation against Cancer, F94 and grants from Flanders Institute for Biotechnology (VIB). PV holds a Methusalem grant (BOF09/01M00709) from the Flemish Government.

1. Houde C, Banks KG, Coulombe N, Rasper D, Grimm E, Roy S et al. Caspase-7 expanded function and intrinsic expression level underlies strain-specific brain phenotype of caspase-3-null mice. J Neurosci 2004; 24: 9977-9984.

2. Choudhury S, Bhootada Y, Gorbatyuk O, Gorbatyuk M. Caspase-7 ablation modulates UPR, reprograms TRAF2-JNK apoptosis and protects T17M rhodopsin mice from severe retinal degeneration. Cell Death Dis 2013; 4: e528.

3. Matalova E, Vanden Berghe T, Svandova E, Vandenabeele P, Healy C, Sharpe PT et al. Caspase-7 in molar tooth development. Arch Oral Biol 2012; 57: 1474-1481.

4. Matalova E, Lesot H, Svandova E, Vanden Berghe T, Sharpe PT, Healy C et al. Caspase-7 participates in differentiation of cells forming dental hard tissues. Dev Growth Differ 2013; 55: 615-621.

5. Lamkanfi M, Festjens N, Declercq W, Vanden Berghe T, Vandenabeele P. Caspases in cell survival, proliferation and differentiation. Cell Death Differ 2007; 14: 44-55.

6. Chung UI, Kawaguchi H, Takato T, Nakamura K. Distinct osteogenic mechanisms of bones of distinct origins. J Orthop Sci 2004; 9: 410-414.

7. Groeneveld EHJ, Burger EH. Bone morphogenetic proteins in human bone regeneration. Eur J Endocrinol 2000; 142: 9-21.

8. Marie PJ. Fibroblast growth factor signaling controlling bone formation: an update. Gene 2012; 498: 1-4.

9. Spinella-Jaegle S, Rawadi G, Kawai S, Gallea S, Faucheu C, Mollat P et al. Sonic hedgehog increases the commitment of pluripotent mesenchymal cells into the osteoblastic lineage and abolishes adipocytic differentiation. J Cell Sci 2001; 114: 2085-2094.

10. Yang $Y Q$, Tan $Y Y$, Wong $R$, Wenden $A$, Zhang $L K$, Rabie $A B$. The role of vascular endothelial growth factor in ossification. Int J Oral Sci 2012; 4: 64-68.

11. Clarkin CE, Gerstenfeld LC. VEGF and bone cell signalling: an essential vessel for communication? Cell Biochem Funct 2013; 31: 1-11.

12. Chen G, Deng C, Li YP. TGF- $\beta$ and BMP signaling in osteoblast differentiation and bone formation. Int J Biol Sci 2012; 8: 272-288.

13. Bendall AJ, Abate-Shen C. Roles for Msx and Dlx homeoproteins in vertebrate development. Gene 2000; 247: 17-31.

14. Wright E, Hargrave MR, Christiansen J, Cooper L, Kun J, Evans T et al. The Sry-related gene Sox9 is expressed during chondrogenesis in mouse embryos. Nat Genet 1995; 9: $15-20$.

15. Komori T. Runx2, a multifunctional transcription factor in skeletal development. J Cell Biochem 2002; 87: 1-8.

16. Diep L, Matalova E, Mitsiadis TA, Tucker AS. Contribution of the tooth bud mesenchyme to alveolar bone. J Exp Zool B Mol Dev Evol 2009; 312B: 510-517.

17. Lungová V, Radlanski RJ, Tucker AS, Renz H, Míšek I, Matalová E. Tooth-bone morphogenesis during postnatal stages of mouse first molar development. J Anat 2011; 218: $699-716$.
18. Crawford ED, Wells JA. Caspase substrates and cellular remodeling. Annu Rev Biochem 2011; 80: 1055-1087.

19. Schwerk C, Schulze-Osthoff K. Non-apoptotic functions of caspases in cellular proliferation and differentiation. Biochem Pharmacol 2003; 66: 1453-1458.

20. Ramaesh T, Bard JB. The growth and morphogenesis of the early mouse mandible: a quantitative analysis. J Anat 2003; 203: 213-222.

21. Kim IS, Otto F, Zabel B, Mundlos S. Regulation of chondrocyte differentiation by Cbfa1. Mech Dev 1999; 80: 159-170.

22. Aghaloo TL, Chaichanasakul T, Bezouglaia O, Kang B, Franco R, Dry SM et al. Osteogenic potential of mandibular versus long-bone marrow stromal cells. J Dent Res 2010; 89: 1293-1298.

23. Kashima TG, Nishiyama T, Shimazu K, Shimazaki M, Kii I, Grigoriadis AE et al. Periostin, a novel marker of intramembranous ossification, is expressed in fibrous dysplasia and in c-Fos-over expressing bone lesions. Hum Pathol 2009; 40: 226-237.

24. Suttapreyasri S, Koontongkaew S, Phongdara A, Leggat U. Expression of bone morphogenetic proteins in normal human intramembranous and endochondral bones. Int J Oral Maxillofac Surg 2006; 35: 444-452.

25. De Spiegelaere W, Cornillie P, Casteleyn C, Burvenich C, Van den Broeck W. Detection of hypoxia inducible factors and angiogenic growth factors during metal endochondral and intramembranous ossification. Anat Histol Embryol 2010; 39: 376-384.

26. Kugimiya F, Kawaguchi H, Kamekura S, Chikuda H, Ohba S, Yano F et al. Involvement of endogenous bone morphogenetic protein (BMP) 2 and BMP6 in bone formation. J Biol Chem 2005; 280: 35704-35712.

27. Musch T, Öz Y, Lyko F, Breiling A. Nucleoside drugs induce cellular differentiation by caspase-dependent degradation of stem cell factors. PLoS One 2010; 5: e10726.

28. Talanian RV, Quinlan C, Trautz S, Hackett MC, Mankovich JA, Banach D et al. Substrate specificities of caspase family proteases. J Biol Chem 1997; 272: 9677-9682.

29. Lakhani SA, Masud A, Kuida K, Porter GA Jr, Booth CJ, Mehal WZ et al. Caspases 3 and 7: key mediators of mitochondrial events of apoptosis. Science 2006; 311: $847-851$.

30. Miura M, Chen XD, Allen MR, Bi Y, Gronthos S, Seo BM et al. A crucial role of caspase-3 in osteogenic differentiation of bone marrow stromal stem cells. J Clin Invest 2004; 114 1704-1713.

31. Nakatsumi $\mathrm{H}$, Yonehara S. Identification of functional regions defining different activity in caspase-3 and caspase-7 within cells. J Biol Chem 2010; 285: 25418-25425.

32. Walsh JG, Cullen SP, Sheridan C, Lüthi AU, Gerner C, Martin SJ. Executioner caspase-3 and caspase-7 are functionally distinct proteases. Proc Natl Acad Sci USA 2008; 105: 12815-12819.

33. Chandler JM, Cohen GM, MacFarlane M. Different subcellular distribution of caspase-3 and caspase-7 following Fas-induced apoptosis in mouse liver. J Biol Chem 1998; 273: 10815-10818.

34. Demon D, Van Damme P, Vanden Berghe T, Vandekerckhove J, Declercq W, Gevaert K et al. Caspase substrates: easily caught in deep waters? Trends Biotechnol 2009; 27: 680-688.

35. Moriishi T, Maruyama Z, Fukuyama R, Ito M, Miyazaki T, Kitaura H et al. Overexpression of $\mathrm{Bcl} 2$ in osteoblasts inhibits osteoblast differentiation and induces osteocyte apoptosis. PLoS One 2011; 6: e27487.

36. Tamburstuen MV, Reseland JE, Spahr A, Brookes SJ, Kvalheim G, Slaby I et al. Ameloblastin expression and putative autoregulation in mesenchymal cells suggest a role in early bone formation and repair. Bone 2011; 48: 406-413.

37. Suzuki S, Haruyama N, Nishimura F, Kulkarni AB. Dentin sialophosphoprotein and dentin matrixprotein-1: two highly phosphorylated proteins in mineralized tissues. Arch Oral Biol 2012; 57: 1165-1175.

38. Korpi JT, Aström P, Lehtonen N, Tjäderhane L, Kallio-Pulkkinen S, Siponen M et al. Healing of extraction sockets in collagenase-2 (matrix metalloproteinase-8)-deficient mice. Eur J Oral Sci 2009; 117: 248-254.

39. Wang M, Jin H, Tang D, Huang S, Zuscik MJ, Chen D. Smad1 plays an essential role in bone development and postnatal bone formation. Osteoarthritis Cartilage 2011; 19 : 751-762.

40. Nohe A, Keating E, Knaus P, Petersen NO. Signal transduction of bone morphogenetic protein receptors. Cell Signal 2004; 16: 291-299.

41. Hellingman CA, Davidson EN, Koevoet W, Vitters EL, van den Berg WB, van Osch GJ et al. Smad signaling determines chondrogenic differentiation of bone-marrow-derived mesenchymal stem cells: inhibition of Smad1/5/8P prevents terminal differentiation and calcification. Tissue Eng Part A 2011; 17: 1157-1167.

42. Wise GE, He H, Gutierrez DL, Ring S, Yao S. Requirement of alveolar bone formation for eruption of rat molars. Eur J Oral Sci 2011; 119: 333-338.

43. Mishina Y, Starbuck MW, Gentile MA, Fukuda T, Kasparcova V, Seedor JG et al. Bone morphogenetic protein type IA receptor signalling regulates postnatal osteoblast function and bone remodeling. J Biol Chem 2004; 279: 27560-27566.

44. Golub EE, Boesze-Battaglia K. The role of alkaline phosphatase in mineralization. Curr Opin Orthop 2007; 18: 444-448.

45. San Miguel SM, Goseki-Sone M, Sugiyama E, Watanabe H, Yanagishita M, Ishikawa I. Tissue-non-specific alkaline phosphatase mRNA expression and alkaline phosphatase activity following application of retinoic acid in cultured human dental pulp cells. Arch Oral Biol 1999; 44: 861-869. 
46. Gianni M, Studer M, Carpani G, Terao M, Garattini E. Retinoic acid induces liver/bone/ kidney-type alkaline phosphatase gene expression in F9 teratocarcinoma cells. Biochem $J$ 1991; 274: 673-678

47. Rambaldi A, Terao M, Bettoni S, Bassan R, Battista R, Barbui T et al. Differences in the expression of alkaline phosphatase mRNA in chronic myelogenous leukemia and paroxysmal nocturnal hemoglobinuria polymorphonuclear leukocytes. Blood 1989; 73: $1113-1115$

48. Kyeyune-Nyombi E, Nicolas V, Strong DD, Farley J. Paradoxical effects of phosphate to directly regulate the level of skeletal alkaline phosphatase activity in human osteosarcoma (SaOS-2) cells and inversely regulate the level of skeletal alkaline phosphatase mRNA. Calcif Tissue Int 1995; 56: 154-159.

49. Zhivotovsky B, Samali A, Gahm A, Orrenius S. Caspases: their intracellular localization and translocation during apoptosis. Cell Death Differ 1999; 6: 644-651.

50. Faleiro L, Lazebnik Y. Caspases disrupt the nuclear-cytoplasmic barrier. J Cell Biol 2000; 151: 951-959.

51. Germain M, Affar EB, D'Amours D, Dixit VM, Salvesen GS, Poirier GG. Cleavage of automodified poly (ADP-ribose) polymerase during apoptosis. Evidence for involvement of caspase-7. J Biol Chem 1999; 274: 28379-28384.

52. Faragher AJ, Sun XM, Butterworth M, Harper N, Mulheran M, Ruchaud S et al. Death receptor-induced apoptosis reveals a novel interplay between the chromosomal passenger complex and CENP-C during interphase. Mol Biol Cell 2007; 18: 1337-1347.
53. Liu X, Zou H, Slaughter C, Wang X. DFF a heterodimeric protein that functions downstream of caspase-3 to trigger DNA fragmentation during apoptosis. Cell 1997; 89: 175-184.

54. Obara N, Lesot $\mathrm{H}$. Subcellular localization of beta-catenin and cadherin expression in the cap-stage enamel organ of the mouse molar. Histochem Cell Biol 2004; 121: 351-358.

55. Duprez L, Takahashi N, Van Hauwermeiren F, Vandendriessche B, Goossens V, Vanden Berghe $T$ et al. RIP kinase-dependent necrosis drives lethal systemic inflammatory response syndrome. Immunity 2011; 35: 908-918.

(c) (i) $(9)$ Cell Death and Disease is an open-access journal published by Nature Publishing Group. This work is licensed under a Creative Commons Attribution-NonCommercialNoDerivs 3.0 Unported License. The images or other third party material in this article are included in the article's Creative Commons license, unless indicated otherwise in the credit line; if the material is not included under the Creative Commons license, users will need to obtain permission from the license holder to reproduce the material. To view a copy of this license, visit http://creativecommons.org/licenses/ by-nc-nd/3.0/ 